\title{
The Large Electrochemical Capacitance of Microporous Doped Carbon Obtained by Using a Zeolite Template
}

\author{
By Conchi O Ania, Volodymir Khomenko, Encarnación Raymundo-Piñero, José B \\ Parra, François Béguin*
}

A novel microporous template carbon doped with nitrogen has been synthesized via a two-step nanocasting process using acrylonitrile and propylene as precursors and $\mathrm{Na}-\mathrm{Y}$ zeolite as scaffold. Liquid phase impregnation and in-situ polymerization of the nitrogenated precursor inside the nanochannels of the inorganic scaffold, followed by gas phase impregnation with propylene, enabled a pore-size control and functionality tuning of the resulting carbon. The material hereby obtained presented a narrow pore size distribution within the micropore range and a large amount of heteroatoms (i.e., oxygen and nitrogen). Besides, the carbon material inherited the ordered structure of the inorganic host. Such features simultaneously present in the carbon turned out in a unique performance of the material as electrode for supercapacitors. Although presenting a moderately developed specific surface area $\left(S_{\mathrm{BET}}=1680 \mathrm{~m}^{2} \mathrm{~g}^{-1}\right)$, the template carbon displays a large gravimetric capacitance $\left(340 \mathrm{~F} \mathrm{~g}^{-1}\right)$ in aqueous medium due to the combined electrochemical activity of the heteroatoms and accessible porosity. This material enables to operate at $1.2 \mathrm{~V}$ in aqueous medium with a good cycleability beyond 10000 cycles, being extremely promising for the development of high energy density supercapacitors.

[*] Prof. F. Béguin, Dr. E. Raymundo-Piñero, Dr. CO. Ania, Dr. V. Khomenko

Research Centre on Divided Matter,

CNRS-University, 1B rue de la Férollerie,

45071 Orléans, France

E-mail: beguin@crns-orleans.fr

Dr. JB. Parra

Energy and Environment Department,

Instituto Nacional del Carbón, CSIC

P.O. 73, Oviedo 33080, Spain

[**] The authors thank Norit for kindly supplying the activated carbon adsorbent. COA thanks MEC, Spain, for the financial support (EX2004-0612). The kindest help of R. Benoit for the XPS data is acknowledged. 


\section{INTRODUCTION}

Energy storage in electrical double layer capacitors (EDLCs) is based on the storage and release of charges along the double layer formed at the electrode/electrolyte interface and it therefore strongly depends on the surface area of this interface. ${ }^{[1]}$ For this reason, activated carbons are widely used as electrode material in supercapacitors. ${ }^{[2]}$ Although a high surface area is usually considered as a main requirement for carbon-based electrodes to be used in EDLCs, a further analysis of the electrochemical behavior in different electrolytic media shows that an adequate pore size is a very critical parameter for obtaining high values of capacitance. ${ }^{[3-5]}$ The essentially microporous and disordered texture of activated carbons hinders their electrochemical performance because the narrow micropores are not easily accessible to the electrolyte and thus do not always contribute to the total double layer capacitance. ${ }^{[4]}$ Moreover, for high degrees of activation, a saturation of capacitance is attained, either because the electrical double layer is not efficiently formed in too wide pores ${ }^{[3]}$ or because the pore walls are too thin. ${ }^{[6]}$ Hence, in order to obtain high values of capacitance, the pore size of the carbon electrode must match the dimensions of the electrolyte used i) to assure the accessibility of the ions to the micropores surface and ii) to avoid the capacitance saturation.

Along with porosity, the electrical conductivity ${ }^{[7]}$ and surface functionalities ${ }^{[2]}$ of the carbon material must be taken into account for the electrochemical performance. It is well-known that heteroatoms can play a critical role in pseudo-faradaic charge transfer reactions, giving rise to pseudo-capacitance effects. ${ }^{[2,7,8]}$ For these reasons, it becomes essential to tune both the porous and chemical features of the carbon materials for their application in supercapacitors.

Many novel approaches to design the porous texture of carbons have been recently developed. ${ }^{[9]}$ Among them, the template carbonization has been considered as a unique and versatile method for providing well-designed and almost full pore-controlled carbon materials. ${ }^{[10,11]}$ The resulting carbons possess a large specific surface area, high porosity and a controlled narrow pore size distribution, these features being not attained by conventional approaches (i.e, physical and chemical activation procedures). Pioneering research studies on this method were performed by Kyotani et al, using layered clay minerals. ${ }^{[12]}$ Later, they extended the research to microporous inorganic frameworks (i.e., zeolites) with different structures and since then many authors have used this 
procedure (more or less successfully) to obtain highly microporous and ordered carbon materials.

Despite the need of such porous carbons for energy storage, major efforts have focused on the preparation, optimization and physico-chemical characterization of template carbons, while scarce results can be found on their electrochemical performance. ${ }^{[13-17]}$ Among these few papers, good electrochemical performance of mesoporous dopedcarbons with somewhat layer-like structure obtained from clay-minerals as templates ${ }^{[17]}$ is reported at low current densities (ca. $20 \mathrm{~mA} \mathrm{~g}^{-1}$ ). However the authors did not investigate the materials at more realistic operating conditions, nor their performance and stability upon long-term cycling. More recently, other nitrogen-enriched mesoporous carbons demonstrated capacitance values ca. $200 \mathrm{~F} \mathrm{~g}^{-1}$ at higher current density. ${ }^{[18,19]}$

In this paper, we present the electrochemical behavior of a template microporous carbon with a narrow pore size distribution used as electrode for supercapacitors in aqueous medium. In order to obtain an advanced material with high values of capacitance, we have used a nitrogen-containing carbon precursor to dope the carbon matrix and favor the development of pseudo-faradaic charge transfer processes. The combination of the template procedure using a zeolitic ordered host and a nitrogen-containing pre-polymer which is polymerized inside the scaffold, enabled to obtain a carbon with a unique combination of high porosity, micropores perfectly adapted to the size of the electrolyte and straight channels enhancing the diffusion of ions to micropores and active surface groups.

\section{RESULTS AND DISCUSSION}

\subsection{Replication of the zeolite framework}

The template procedure used in this work was inspired from the double infiltration method described by Ma et al in the literature. ${ }^{[10]}$ In a first step, the nitrogen rich carbon precursor (acrylonitrile) was introduced into the pores of the dried zeolite under reduced pressure at room temperature, and it was allowed to polymerize within the nanochannels of the inorganic framework. In the second step, the resultant zeolite/polymer composite was heated up to $700^{\circ} \mathrm{C}$ under inert atmosphere and then chemical vapor deposition (CVD) under propylene flow was carried out. The material was further heated at $900^{\circ} \mathrm{C}$ 
under $\mathrm{N}_{2}$ flow before etching the zeolite. According to the literature, this thermal stabilization allows obtaining a material with a rigid and ordered structure; ${ }^{[10]}$ otherwise, even if the zeolite channels are fully filled, the linkages between carbon clusters are easily broken during etching of the inorganic framework.

As a result, the XRD pattern of the carbon replica (Y-AN) shows a peak at $6^{\circ}$ proving that the structural regularity of zeolite has been well replicated (Figure 1). This is in good agreement with other reports ${ }^{[10]}$ on the use of Na-Y zeolite as host. A broad peak at $25^{\circ}$ ascribed to the stacking structure of graphitic layers was also obtained; since this feature was not observed in the composites, it is clear that it arises from the stacking of the structure during the etching process and not from the deposition of pyrolytic carbon on the external surface during CVD. ${ }^{[21,22]}$ In sum, the Y-AN carbon presents a structural periodicity, as opposed to common activated carbons.

Table 1 compiles details of the textural characteristics of the template carbon. For clarity, the nitrogen adsorption isotherms are shown in Figure 2. The $\mathrm{N}_{2}$ adsorption data reveal that this carbon is highly porous, the pore volume being higher than that of the pristine zeolite $\left(0.370 \mathrm{~cm}^{3} \mathrm{~g}^{-1}\right)$. The porosity originates from the structure of the $\mathrm{Y}$ zeolite, where the framework in the carbon/zeolite composite turns into the pores of the resulting carbon after etching the inorganic matrix. It has to be stressed that the surface area is much higher than that corresponding to the char obtained when the carbon precursor (polyacrylonitrile) is carbonized under the same conditions but in absence of the zeolitic framework, which hardly presents any porosity. ${ }^{[23]}$ In such a case, a second step of activation by traditional methods (physical or chemical activation) is mandatory to develop the porosity of the material. ${ }^{[24]}$ Even after activation with $50 \%$ burn-off degree of the carbonized polymeric precursor, the BET specific surface area remains much lower than that obtained for the template carbon. ${ }^{[8,24,25]}$

It should be mentioned that the relatively low specific surface area of this carbon - if compared to highly microporous carbons with up to $3500 \mathrm{~m}^{2} \mathrm{~g}^{-1}$ obtained from a twostep impregnation using furfuryl alcohol ${ }^{[10]}$ - is attributed to a partial infiltration of the host when an aqueous solution of the carbon precursor is employed. As discussed elsewhere, ${ }^{[26]}$ this leads to an incomplete filling of the framework, and therefore unfilled pores coalesce during carbonization, which has a detrimental effect on porosity development.

On the other hand, earlier works on the preparation of templated carbons from $\mathrm{Na}-\mathrm{Y}$ zeolite and using only PAN as carbon precursor have not succeeded to obtain a carbon 
with the same features as the one hereby described. ${ }^{[20,27]}$ The obtained carbons present much lower surface areas and low micropore and mesopore volumes. In the case of YAN, the double-impregnation procedure (i.e., liquid impregnation + CVD) is beneficial, being responsible for a better filling of the zeolite channels.

For obtaining more information on porosity, the pore size distribution (PSD) curves were determined by the application of the density functional theory (DFT) method to the $\mathrm{N}_{2}$ adsorption isotherms, assuming slit-shape geometry of the pores. As the thickness of the zeolite framework is around $1 \mathrm{~nm}^{[10]}$, one might expect a microporous carbon with a pore size equivalent to the dimensions of the zeolite walls (i.e., $1 \mathrm{~nm}$ ). The PSD obtained by DFT (Figure 3) shows a maximum located at $1.2 \mathrm{~nm}$, along with a small contribution of narrow micropores (i.e., $\mathrm{w}<0.7 \mathrm{~nm}$ ). In fact, this maximum is always located at the same position in carbons obtained using zeolite $\mathrm{Y}$ as template, regardless the carbon precursor or the experimental conditions, ${ }^{[10,11,27]}$ proving that it comes from the replication of the zeolite. The main difference between the samples settles on the height of the maximum (i.e., the total amount of micropores) and the volume of mesopores created, as also evidenced by other authors. ${ }^{[21,27]}$ When the channels and cavities of the zeolite are fully filled, the PSD is almost exclusively composed of $1.2 \mathrm{~nm}$ micropores, ${ }^{[11]}$ incomplete impregnation leading to the development of mesopores and lower volume of micropores (lower height of the PSD maximum).

Understanding why the maximum is located at $1.2 \mathrm{~nm}$ instead of the expected at $1 \mathrm{~nm}$, is a tough task and still remains controversial. Some hypothesis claims that the slit-pore shape model used by the DFT might not be applicable to these template carbons, ${ }^{[11]}$ whereas a recent paper by Roussel et al. ${ }^{[28]}$ reports that even though the assumed pore model does not fully agree with the expected one based on the structure of the zeolite, the obtained PSD provides a reasonable representation of the template carbons. Notwithstanding this issue was not the scope of this work, in sum we could assume that the template carbon possess a narrow pore size distribution centered within 1-1.2 nm. For the sake of comparison, a commercial activated carbon (AC) supplied by Norit and intended for supercapacitor applications was also studied and its porosity and electrochemical performance hereby reported. It can be seen that both materials possess, a priori, the same porosity with similar pore volumes, ratio meso/micro and total surface area (Table 1). However, if the PSD is evaluated for both carbons, a great difference can be observed (Figure 3). The commercial carbon (AC) shows the features usually found 
in activated carbons obtained from traditional methods; this is, a broad distribution which diverges from the narrow one in the template carbon. For instance, the volume of pores within the range $1-1.5 \mathrm{~nm}$ accounts for $60 \%$ of the total pore volume for the template carbon, whereas it represents only a $23 \%$ in the commercial carbon.

In order to obtain a material with comparable porosity but without $\mathrm{N}$-doping of the carbon matrix, a sample (Y-Ac) was prepared by a template route using acetylene as carbon precursor and a one-step infiltration step. In this regard, one should bear in mind that when dealing with templated carbons, the porous texture depends to a large extent on the carbon precursor employed (ca. polymerization pathway). ${ }^{[10]}$ Although it is not an easy task, we have successfully prepared a carbon possessing a rather similar porous texture: narrow PSD within the micropore range (Figure 3) inherited from the inorganic host, along with a slightly higher contribution of mesopores (Table 1). For the purpose of illustrating the effect of the N-doping, the electrochemical behaviour of this carbon was also investigated (see discussion below) and compared to that in commercial and N-enriched microporous carbons.

In sum, an N-doped carbon with a precisely controlled microporosity in a narrow pore range, together with a large surface area and pore volume is obtained in the present work by using a zeolite as template, which is not possible from conventional activation methods.

\subsection{Chemical features of the template carbons}

The elemental analysis of the Y-AN template carbon revealed relatively large amounts of nitrogen and oxygen, yielding the following weight fractions: $\mathrm{C}=83.6 \%, \mathrm{~N}=6.0 \%$, $\mathrm{H}=2.7 \%, \mathrm{O}=7.7 \%$. Oxygen is commonly present in PAN-derived carbons ${ }^{[23,24]}$ as a consequence of its incorporation to the dangling bonds when the material is exposed to air. In our case, it might also come due to the oxidation of the interlayer carbon by the inorganic host during the stabilization treatment at high temperature $\left(900^{\circ} \mathrm{C}\right)$.

In the case of nitrogen-doped activated carbons, the amount of nitrogen strongly depends on the carbonization temperature of the precursor and on the degree of char activation. Obtaining carbons with large nitrogen content and, at the same time, high porous texture is not an easy task. If the carbon is highly activated to obtain the desired porosity, an unavoidable detriment in the nitrogenated functionalities comes out. ${ }^{[25]} \mathrm{By}$ contrast, the Y-AN carbon herein reported exhibits both craved properties, due to the benefits of the template procedure. Despite the high stabilization temperature $\left(900^{\circ} \mathrm{C}\right)$, 
the thermal heating carried out in the zeolite/carbon composite seems to prevent the removal of nitrogen while keeping the porous texture.

Su et al. ${ }^{[27]}$ have reported nitrogen-doped template carbons using the ammonium-form of the $\mathrm{Y}$ zeolite as both the host and the nitrogen carrier, which enabled the incorporation of nitrogen to the carbon matrix, along with high specific surface area. However, the carbon exhibit somewhat broad PSD, and the regularity of the zeolite was not transferred to the porous carbons. Consequently, a highly porous PAN-derived carbon has been prepared exhibiting at the same time the characteristics of high surface area, controlled and narrow pore size distribution in the microporous range, and high content of heteroatoms (i.e., nitrogen and oxygen). These unique characteristics make the carbon extremely attractive for its use as electrode material in supercapacitors.

XPS surface analysis of the template carbon showed comparable concentration values of nitrogen and oxygen to those determined for the bulk. In the case of oxygen, it indicates that functionalities are incorporated to the matrix and cannot be considered as oxygen chemisorbed on the carbon surface. The deconvolution of the XPS spectra enabled to identify the surface functionalities. The N1s core level was fitted by 3 peaks whose position and assignment ${ }^{[29]}$ are given in Table 2. Quaternary (N-Q) and pyridinic (N-6) functionalities are dominant in the carbon, although a contribution of pyridine Noxide cannot be neglected $(\mathrm{N}-\mathrm{X})$. A relevant feature is that the ratio of nitrogen located inside (N-Q) or at the edges (N-6, N-X) of the graphene layers is almost 1:1, which is due to the high temperature at which the sample has been stabilized. The O1s spectrum was assigned to oxygen doubly bonded to carbon (quinone-type groups) and singly bonded in phenol and ether aromatic structures (Table 2).

The composition of the Y-Ac sample is also compiled in Table 2. The carbon presents a high amount of oxygen (ca. $7.1 \mathrm{wt}$ \% as evaluated by elemental analysis), as most carbons obtained from template routes. The O1s spectrum indicates the presence of mainly phenol, ether groups and quinone on the carbon surface, similarly to the oxygencontaining groups in the N-doped Y-AN carbon.

\subsection{Electrochemical behavior}

Having the properties of large surface area and ordered structure given by the nanocasting of the zeolite, along with the presence of a priori electroactive species (as a result of the heteroatoms incorporated to the carbon matrix), the Y-AN material is extremely interesting to be investigated as electrode for supercapacitors. 
The electrochemical performance of the template Y-AN carbon was firstly analyzed by using a two electrode cell and $1 \mathrm{~mol} \mathrm{~L}^{-1} \mathrm{H}_{2} \mathrm{SO}_{4}$ as electrolyte. Cyclic voltammograms of Y-AN, Y-Ac and AC samples are compared in Figure 4. The first difference between the carbons concerns the shape of the voltammograms. The quite rectangular shape in the case of AC is indicative of a pure capacitive behavior in this voltage range. By contrast, the Y-AN template material showed a wide reversible hump which points out a large pseudo-faradaic contribution to the overall performance linked to the surface functionalities. Similar shapes, deviated from the rectangular one, have been reported in materials modified by oxidation. ${ }^{[30]}$ An extremely high value of gravimetric capacitance is attained for $\mathrm{Y}$-AN as compared to $\mathrm{AC}$ (340 vs $120 \mathrm{~F} \mathrm{~g}^{-1}$, respectively). Notwithstanding the fact that both carbons present comparable specific surface area, total pore volume and micropore volume (Table 1), the overall capacitance is almost 3 times higher in the template carbon. Although the wettability of the carbon might play some role, this large increase is mainly related to an additional pseudo-faradaic contribution to the capacitance. ${ }^{[1,2,8,18]}$ Whereas literature essentially emphasizes the relationship between the texture of nanoporous carbons and their capacitance properties, particularly the surface area, ${ }^{[31]}$ it is demonstrated here that electroactive surface functionalities do have a crucial role.

For the purpose of better illustrating the effect of the N-doping, the electrochemical behaviour of Y-AN was compared to that of Y-Ac. As shown in Figure 4, the Nenriched carbon shows a superior electrochemical performance, with 1.5 times higher value of capacitance (340 vs $240 \mathrm{~F} \mathrm{~g}^{-1}$ for Y-AN and Y-Ac, respectively). As both carbons possess somewhat similar porosity and oxygen content, this result suggests that the presence of nitrogen in the carbon matrix is responsible for an important pseudocapacitive contribution. The implication of nitrogen in pseudo-faradaic effects has already been studied in the literature although the mechanism still remains somewhat uncertain. $^{[1,18,19,23-25]}$

In order to explore the pseudo-capacitive contribution in the acidic electrolytic medium, cyclic voltammetry has been recorded for Y-AN and AC in a three electrode cell using a graphite rod as counter electrode and the $\mathrm{Hg} / \mathrm{Hg}_{2} \mathrm{SO}_{4}$ reference electrode (Figure 5). By comparison with AC, remarking the wide humps on the voltammogram for the template carbon, it is evident that reversible redox transitions involving proton exchange with the electrolyte are occurring while the Y-AN electrode is polarized. The waves are rather poorly shaped and spread over a wide potential range (from -0.5 to $+0.2 \mathrm{~V}$ vs 
$\mathrm{Hg} / \mathrm{Hg}_{2} \mathrm{SO}_{4}$ ). Due to the various functionalities in the material, the width of these peaks suggests that various redox processes overlap. It is known that the reduction potential of the quinone/hydroquinone pair shifts down to values close to $-0.15 \mathrm{~V}$ vs $\mathrm{Hg} / \mathrm{Hg}_{2} \mathrm{SO}_{4}$ in carbons; ${ }^{[32]}$ hence taking into account the XPS data, we partially attribute the cathodic peak to the reduction of quinone-like groups, whereas the anodic wave is assigned to the oxidation of hydroquinone-like groups in different environments. ${ }^{[33]}$ More recently it has been shown that pyrone-type structures (combination of non-neighboring carbonyl and ether-oxygen atoms at the edge of the graphene layers) can accept two protons and two electrons in the same range of electrochemical potential as the quinonehydroquinone pair. ${ }^{[34]}$ As for the nitrogen functionalities, a number of mechanisms have been proposed as responsible for the enhancement of capacitance values. ${ }^{[8,18]}$ Hence, Figure 5 suggests that the electrochemical performance of the Y-AN template carbon is due both to charging the electrical double layer and to pseudo-faradaic redox reactions involving the nitrogenated and oxygenated functionalities.

The performance of supercapacitors built from Y-AN and AC was evaluated at different values of maximum voltage by galvanostatic charge-discharge (Figure 6). Whereas the charge-discharge branches of AC are linear (which is indicative of a capacitive response), the pseudo-capacitive behavior is confirmed for the template carbon by their non-linear shape. The discharge capacitance increases with voltage and the coulombic efficiency -calculated as the ratio between the total amount of discharge and charge of the capacitor obtained from the galvanostatic experiments- is still considerably high at $1.2 \mathrm{~V}$ (95\%). Beyond $1.2 \mathrm{~V}$, a marked curvature appears in the galvanostatic charge branch at high voltage value, indicating that the template carbon starts to be oxidized. On the contrary, the AC carbon could only operate at a maximum voltage of $0.8 \mathrm{~V}$; above this value, the capacitor collapsed due to oxidation, and thus damage of the carbon structure. ${ }^{[35]}$ In summary, the capacitor built from the Y-AN template carbon seems to be able to operate at $1.2 \mathrm{~V}$, which is much higher than the usual operating voltage for symmetric systems based on carbon electrodes in aqueous medium. ${ }^{[36]}$ The closest behavior that can be found in the literature corresponds to non porous carbons obtained by carbonization of high oxygen content biopolymers, ${ }^{[37]}$ which are able to operate at $1.0 \mathrm{~V}$ with a good cycleability.

To corroborate this result, the performance of capacitors built with Y-AN was further investigated by galvanostatic long-term cycling (up to $10^{4}$ cycles) at two current loads. Figure 7 shows the evolution of capacitance of the device charged at 0.6 and $1.2 \mathrm{~V}$ as a 
function of the number of cycles. With a maximum voltage of $0.6 \mathrm{~V}$ and a current density of $100 \mathrm{~mA} \mathrm{~g}^{-1}$, the material shows an acceptable cycle life with high capacitance values of $300 \mathrm{~F} \mathrm{~g}^{-1}$ after a relatively high number of cycles (higher than 2500 cycles). After $10^{4}$ cycles, the capacitance is still as high as $260 \mathrm{~F} \mathrm{~g}^{-1}$. Under the heavy conditions of $1.2 \mathrm{~V}$ in aqueous medium and $200 \mathrm{~mA} \mathrm{~g}^{-1}$, the capacitance dependence remains parallel to the plot obtained at $100 \mathrm{~mA} \mathrm{~g}^{-1}$, confirming still a satisfactory behavior of the material. As expected for a material with pseudo-capacitance properties, the values of capacitance at a current density of $200 \mathrm{~mA} \mathrm{~g}^{-1}$ are slightly lower than at $100 \mathrm{~mA} \mathrm{~g}^{-1}$. By contrast, the carbon AC could not be cycled at a voltage of $1 \mathrm{~V}^{2}$ and $100 \mathrm{~mA} \mathrm{~g}^{-1}$; after a few tens of cycles the capacitor collapsed (Figure 7, inset), likely due to the carbon damage provoked by its oxidation and/or evolution of gases produced by electrolyte decomposition. At a voltage of $0.6 \mathrm{~V}$, the cycleability of AC is satisfactory, but the capacitance after $10^{4}$ cycles is only $115 \mathrm{~F} \mathrm{~g}^{-1}$.

In this regard, several authors have stated that the detriment in the cycle life of capacitors might be related to the surface groups, ${ }^{[38]}$ because of changes arising in the functionalities while charging/discharging the device. We have investigated this issue by analyzing the surface composition of Y-AN by XPS after 10000 cycles at $0.6 \mathrm{~V}$. After cycling the capacitor, the electrolyte was washed out and the binder removed from the pellets. The results are shown in Table 2. It can be seen that the amount and proportion of nitrogen-containing groups remains stable. This provides evidence that the faradaic reactions based on nitrogenated functionalities would be fully reversible with cycling. As for the oxygen content, there is an increase from 8 up to $11 \%$ (wt.) which proves that, even when operating at $0.6 \mathrm{~V}$ and $100 \mathrm{~mA} \mathrm{~g}^{-1}$, the material undergoes a slight oxidation with cycling. The oxygenated groups incorporated to the carbon surface are of the same nature as those initially present, i.e., single and double carbon-oxygen bonds; however the proportion of C-O bonds slightly increased.

Previous researches have concluded that the optimized effective pore size for an efficient formation of the EDL depends on the electrolyte used, with pores between 0.7-1 nm leading to higher specific capacitance for acidic medium. ${ }^{[3]}$ In this case, Y-AN has most of the pores at $1.2 \mathrm{~nm}$ which is slightly beyond the optimum value reported, notwithstanding the capacitance is extremely high. Undoubtedly, pseudo-capacitive effects dramatically contribute to the high value of capacitance.

The Y-AN carbon presents a superior performance when compared with both nitrogenenriched mesoporous carbons obtained from template procedures ${ }^{[17,19]}$ or microporous 
carbons obtained from conventional activation methods. ${ }^{[8,13,21]}$ Data from the literature for N-doped mesoporous carbons show capacitance values up to $220 \mathrm{~F} \mathrm{~g}^{-1}$ in sulphuric acid medium. ${ }^{[17-19]}$ Despite containing comparable or higher nitrogen and/or oxygen contents, their lower capacitance highlights that a narrow PSD within the mesopore range (ca. $29 \mathrm{~nm}, 3 \mathrm{~nm}$ ) does not provide an efficient formation of the EDL. This undoubtedly provides a clear evidence of the role of pore size, highlighting the importance of an adequate PSD within the micropore range for energy storage purposes. On the other hand, microporous carbons with similar nitrogen contents and obtained from conventional activation methods ${ }^{[8,13,39]}$ also present inferior electrochemical performance in sulfuric acid (ranging from 127-200 $\mathrm{F} \mathrm{g}^{-1}$ ). Consequently, our results point out the synergetic effects of nitrogen doping and narrow microporous texture on the electrochemical performance of Y-AN.

The higher contribution of pseudo-capacitance should also be linked to the ordered structure of $\mathrm{Y}-\mathrm{AN}$, as shown by the XRD patterns. Compared to the majority of disorganized activated carbons, where the electrolyte must follow tortuous voids to reach the electrochemically active surface, the ordered structure of Y-AN might enhance the connectivity of the pores and accessibility of the electrolyte to surface. Hence, this material presents an effective use of its redox functionalities involved in the pseudo-faradaic phenomena, ensuring a complete utilization of the exposed surfaces for charge storage. This result confirms the paramount importance of not only the pore size and pseudocapacitive effects, but also the pore shape and tortuosity of carbons on their electrochemical performance.

To summarize, the template carbon $\mathrm{Y}$-AN shows a large gravimetric capacitance, with a much better performance than other doped carbons reported in the literature and than a commercial carbon designed for this specific application. The outstanding properties of this carbon allowed achieving a high voltage range in aqueous medium (1.2 V) with large values of capacitance (310 $\mathrm{F} \mathrm{g}^{-1}$ ) over a large number of cycles. Moreover, the maximum energy which could be extracted from the capacitor is 2 and 4 times higher (when operating at 0.6 and $1.2 \mathrm{~V}$, respectively) than that on a commercial carbon-based capacitor (AC) in aqueous medium.

\section{CONCLUSIONS}


We have synthesized a highly porous doped-carbon with a regular structure using a microporous zeolite as template. To the best of our knowledge, this is the first time that a PAN-derived carbon is prepared with the characteristics of high porosity and surface area, controlled and narrow pore size distribution in the microporous range, large heteroatoms content (i.e., nitrogen and oxygen) and accessibility to surface groups. The unique combination of textural and chemical characteristics of this material makes it an excellent one to be used as electrode in supercapacitors.

Two contributions are involved in the charge storage mechanism: a capacitive contribution due to the formation of the electrical double layer, and a pseudo-capacitive effect due to the presence of nitrogenated and oxygenated functionalities that undergo electron transfer reactions in the acidic electrolytic medium used.

The superior performance of this carbon is associated with its adequate pore size distribution and ordered structure, only achieved by the replication of the zeolite. Such properties enable an efficient use of the pseudo-capacitance phenomena, due to the easy diffusion of ions in the straight channels which enhances their access to the bulk of the active material. This result confirms the paramount importance of not only the pore size and pseudocapacitive effects, but also the pore shape and tortuosity on the electrochemical performance of carbons.

Taking profit of the characteristics of the $\mathrm{N}$-doped template carbon, a practical cell voltage of $1.2 \mathrm{~V}$ could be reached in aqueous medium. The capacitance of the supercapacitor is rather stable after a long term charge/discharge cycling ( $10^{4}$ cycles) at heavy regime $\left(1.2 \mathrm{~V}\right.$ and $\left.200 \mathrm{~mA} \mathrm{~g}^{-1}\right)$. The large gravimetric capacitance of the material, along with the high operating voltage reached turn out in a capacitor with energy densities close to the values obtained with electric double layer capacitors working in organic electrolytes.

\section{EXPERIMENTAL}

Materials: $\mathrm{NaY}$ zeolite was obtained from Zeolyst International (13 \% wt. $\mathrm{Na}_{2} \mathrm{O}$, $\mathrm{SiO}_{2} / \mathrm{Al}_{2} \mathrm{O}_{3}$ ratio $\left.=5.1\right)$. Reagent grade acrylonitrile and $\mathrm{HF}(48 \%)$ were purchased from Sigma Aldrich. The commercial activated carbon (AC) was kindly provided by Norit.

Synthesis of the template carbon: Our synthesis method is inspired from that of Ma et al. ${ }^{[10]}$ and is based on a liquid phase impregnation of the inorganic framework and insitu polymerization of the carbon precursor inside the nanochannels of the zeolite, followed by a second step of CVD in gas phase. Acrylonitrile was used as carbon precursor. Briefly, the zeolite is outgassed overnight, and then a mixture of $150 \mathrm{~g}$ of distilled water and $11 \mathrm{~g}$ of acrylonitrile is added under reduced pressure, stirred for 10 
min, and heated at $40^{\circ} \mathrm{C}$. Potassium persulfate $(0.15 \mathrm{~g})$ and $0.075 \mathrm{~g}$ of sodium metabisulfite dissolved in $10 \mathrm{~g}$ of water was added drop wise into the zeolite/AN mixture and stirred for $3 \mathrm{~h}$. The encapsulated product was filtered off, washed in water and dried at $60^{\circ} \mathrm{C}$. After the impregnation, the precursor/silica composite was heated up to $700^{\circ} \mathrm{C}$ under nitrogen atmosphere, and then CVD was performed with propylene (2\% in $\mathrm{N}_{2}$ ) for 4 hours at a flow rate of $150 \mathrm{ml} \mathrm{min}^{-1}$. The sample was further stabilized at $900^{\circ} \mathrm{C}$ for 3 hours under nitrogen. The silica matrix was etched using hydrofluoric acid (48\%) at room temperature, and the resulting template carbon was washed with concentrated $\mathrm{HCl}$ at $60^{\circ} \mathrm{C}$ and distilled water to remove traces of insoluble fluoride salts. The dried product is labeled Y-AN. For the sake of comparison, a second sample was prepared by the template route, using acetylene as carbon precursor in one-step infiltration of the zeolite (CVD, 4 hours). The sample is labeled Y-Ac.

Electrochemical characteristics: Electrodes (mass 8-15 mg) were pressed from a mixture of porous carbon (85\%), polyvinylidene fluoride PVDF (10\%) and carbon black (5\%). Two electrode capacitors were built with a glassy fibrous separator and gold current collectors, using a teflon Swagelok ${ }^{\circledR}$ type system. Aqueous 1 mol.L ${ }^{-1} \mathrm{H}_{2} \mathrm{SO}_{4}$ was used as electrolytic solution. The gravimetric capacitance -C- expressed in farads per gram of carbon material $\left(\mathrm{F} \mathrm{g} \mathrm{g}^{-1}\right)$ was estimated by voltammetry at a scan rate of 2-10 mV $\mathrm{s}^{-1}$ and galvanostatic charge/discharge at a current density of $100-200 \mathrm{~mA} \mathrm{~g}^{-1}$ using a VMP (Biologic, France) multichannel generator. Experiments were also realized with teflon three-electrode cells by using the porous carbon as working electrode, a graphite rod as counter electrode and $\mathrm{Hg} / \mathrm{Hg}_{2} \mathrm{SO}_{4}$ as the reference electrode.

Chemical analysis and textural characterization: The elemental composition (CHNS) was determined using a LECO CHNS-932 analyzer, and the oxygen content was directly determined using a LECO VTF-900 instrument. The porous texture of the activated carbons was characterized by nitrogen adsorption at $-196{ }^{\circ} \mathrm{C}$ (ASAP 2010M, Micromeritics). Before the experiments, the samples were outgassed under vacuum at $120^{\circ} \mathrm{C}$ overnight. The isotherms were used to calculate the specific surface area, $\mathrm{S}_{\mathrm{BET}}$, total pore volume, $\mathrm{V}_{\mathrm{T}}$, and pore size distributions, evaluated using the density functional theory (DFT) assuming a slit-shape pore model. ${ }^{[40]}$ The different types of functional groups were identified by X-ray photoelectron spectroscopy (XPS) in an ESCALAB MK2 (VG-Instrument) apparatus using Mg Ka radiation. In the case of the samples after cycleability, the electrolyte and the binder were removed from the pellets using water and acetone, respectively, and dried before the analysis.

\section{REFERENCES}

[1] a) B. E. Conway, in Electrochemical Supercapacitors: Scientific Fundamentals and Technological Applications. Kluwer Academic/Plenum Publishers, New York, USA, 1999.b) R. Kötz, M. Carlen, Electrochim. Acta 2000, 45, 2483.

[2] E. Frackowiak, F. Béguin, Carbon 2001, 39, 937.

[3] a) E. Raymundo-Piñero, K. Kierzek, J. Machnikowski, F. Béguin, Carbon 2006, 44, 2498. b) J. Chmiola G. Yushin, R. Dash, Y. Gogotsi, J. Power Sources 2006, $158,765$.

[4] a) G. Salitra, A. Soffer, L. Eliad, Y. Cohen, D. Aurbach, J. Electrochem. Soc. 2000, 147, 2486.b) L. Eliad, G. Salitra, A. Soffer, D. Aurbach, J. Phys. Chem. B 2002, 106, 10128. 
[5] Y. J. Kim, Y. Horie, S. Ozaki, Y. Matsuzawa, H. Suezaki, C. Kim, N. Miyashita, M. Endo, Carbon 2004, 42, 1491.

[6] O. Barbieri, M. Hahn, A. Herzog, R. Kötz, Carbon, 2005, 43, 1303.

[7] a) D. Qu, H. Shi, J. Power Sources 1998, 74, 99. b) C. T. Hsieh, H. Teng, Carbon 2002, 40, 667. c) P-Z. Cheng, H. Teng, Carbon 2003, 41, 2057.

[8] a) E. Frackowiak, G. Lota, J. Machnikowski, C. Vix-Guterl, F. Béguin, Electrochim. Acta 2006, 51, 2209. b) K. W. Leitner, B. Gollas., M. Winter, J.O. Besenhard, Electrochim. Acta 2004, 50, 199.

[9] T. Kyotani, Carbon 2000, 38, 269.

[10] Z. Ma, T. Kyotani, Z. Liu, O. Teresaki, A. Tomita, Chem. Mat. 2001, 13, 4413.

[11] a) P-X. Hou, T. Yamazaki, H. Orikasa, T. Kyotani , Carbon 2005, 43, 2624. b) K. Matsuoka, Y. Yamagishi, T. Yamazaki, N. Setoyama, A. Tomita, T. Kyotani, Carbon 2005, 43, 876.

[12] T. Kyotani, N. Sonobe, A. Tomita, Nature 1988, 331, 331.

[13] M. Kodama, J. Yamashita, Y. Soneda, H. Hatori, S. Nishimura, K. Kamegawa, Mat. Sci. Eng. B 2004, 108, 156.

[14] G. Liu, F. Kang, B. Li, Z. Huang, X. Chuan, J. Phys. Chem. Solids, 2006, 67, 1186.

[15] C. Vix-Guterl, E. Frackowiak, K. Jurewicz, M. Friebe, J. Parmentier, F. Béguin, Carbon 2005, 43, 1293.

[16] J. Lee, S. Yoon, T. Hyeon, S.M. Oh, K.B. Kim, Chem. Commun. 1999, 2177.

[17] a) D. Hulicova, M. Kodama, H. Hatori, Chem. Mater. 2006, 18, 2318. b) D. Hulicova, J. Yamashita, Y. Soneda, H. Hatori, M. Kodama, Chem. Mater. 2005, $17,1241$.

[18] W. Li, D. Chen, Z. Li, Y. Shi, Y. Wan, J. Huang, J. Yang, D. Zhao, Z. Jiang, Electrochem. Comm. 2007, 9, 569.

[19] M. Kodama, J. Yamashita, Y. Soneda, H. Hatori, K. Kamegawa, I. Moriguchi, Chem. Lett. 2006, 35, 680.

[20] a) T. Kyotani, T. Nagai, S. Inoue, A. Tomita, Chem. Mater. 1997, 9, 609-615. b) C. J. Meyers, S. D. Shah, S. C. Patel, R. M. Sneeringer, C. A. Bessel, N. R. Dollahon, R. A. Leising, E. S. Takeuchi, J. Phys. Chem. B 2001, 105, 2143.

[21] S. A. Johnson, E. S. Brigham, P. J. Ollivier, T. E. Malouk, Chem. Mat. 1997, 9, 2448.

[22] a) Z. Yang, Y. Xia, R. Mokaza, Microp. Mesop. Mater. 2005, 86, 69. b) F. Su, J. Zeng, Y. Yu, L. Lv, J. Yang Lee, X.S. Zhao, Carbon 2005, 43, 2366.

[23] F. Béguin, K. Szostak, G. Lota, E. Frackowiak, Adv. Mater. 2005, 17, 2380.

[24] J. Machnikowski, B. Grzyb, H. Machnikowska, J.V. Weber, Micro. Meso. Mat. 2005, 82, 113.

[25] K. Jurewicz, K. Babel, A. Ziolkowski, H. Wachowska, J. Phys. Chem. Solids 2004, 65, 269.

[26] C. O. Ania, T. J. Bandosz, Micro. Meso. Mater. 2006, 89, 315. 
[27] F. Su, X. S. Zhao, L. Lv, Z. Zhou, Carbon 2004, 42, 2821.

[28] T. Roussel, J. Jagiello, R. J. M. Pellenq, M. Thommes, C. Bichara, Mol. Simulation 2006, 32, 551.

[29] J. R. Pels, F. Kapteijn, J. A. Moulijn, Q. Zhu, K. M. Thomas, Carbon 1995, 33, 1641.

[30] C-C. Hu, C-C. Wang, J. Power Sources 2004, 125, 299.

[31] H. Shi, Electrochim. Acta 1996, 41, 1633.

[32] K. Kinoshita, in Carbon: Electrochemical and Physicochemical Properties. John Wiley \& Sons, New York, USA, 1988, p. 293-387.

[33] S. Biniak, A. Swiatkowski, M. Pakula, in Chemistry and Physics of Carbon, Vol. 27 (Ed: L.R. Radovic), Dekker, New York, USA, 2001, p. 125-225.

[34] M. A. Montes-Moran, D. Suarez, J. A. Menendez, E. Fuente, Carbon 2004, 42, 1219.

[35] J. Niu, W. G. Pell, B.E. Conway, J. Power Sources 2006, 156, 725.

[36] A. Burke, J. Power Sources 2000, 91, 37.

[37] E. Raymundo-Piñero, F.Leroux, F. Béguin. Adv. Mat, 2006, 18, 1877.

[38] a) K. Kierzek, E. Frackowiak, G. Lota, G. Gryglewicz, J. Machnikowski, Electrochim. Acta 2004, 49, 515. b) M. J. Bleda-Martinez, J. A. Macia-Agullo, D. Lozano-Castello, E. Morallon, D. Cazorla-Amoros, A. Linares-Solano, R. Carbon 2005, 43, 2677.

[39] G. Lota, B. Grzyb, H. Machnikowsk, J. Machnikowski, E. Frackowiak, Chem. Phys. Lett. 2005, 404, 58.

[40] J. P. Olivier, J. Porous Mat. 1992, 5, 9. 
Table 1. Porosity parameters obtained from the $\mathrm{N}_{2}$ adsorption isotherms at $-196{ }^{\circ} \mathrm{C}$ for the templated samples (Y-AN and Y-Ac) and the commercial activated carbon (AC).

\begin{tabular}{lccccc}
\hline Sample & $\begin{array}{c}\mathrm{S}_{\mathrm{BET}} \\
{\left[\mathrm{m}^{2} \mathrm{~g}^{-1}\right]}\end{array}$ & $\begin{array}{c}\mathrm{V}_{\text {TOTAL }} \\
{\left[\mathrm{cm}^{3} \mathrm{~g}^{-1}\right]}\end{array}$ & $\begin{array}{c}\mathrm{V}_{\text {micro }} \\
{\left[\mathrm{cm}^{3} \mathrm{~g}^{-1}\right]}\end{array}$ & $\begin{array}{c}\mathrm{V}_{\text {meso }} \\
{\left[\mathrm{cm}^{3} \mathrm{~g}^{-1}\right]}\end{array}$ & $\begin{array}{c}\text { Ratio } \\
\text { micro/meso }\end{array}$ \\
\hline Y-AN & 1680 & 0.860 & 0.510 & 0.101 & 5.2 \\
AC & 1653 & 0.800 & 0.500 & 0.113 & 4.4 \\
Y-Ac & 1814 & 1.030 & 0.508 & 0.211 & 2.4 \\
\hline
\end{tabular}


Table 2. Data obtained by fitting the N1s and O1s core level spectra of the templated carbon (Y-AN and Y-Ac), before and after 10000 cycles in a capacitor.

\begin{tabular}{lcccccccc}
\hline & N-Q & N-X & N-6 & C-O & C=O & $\begin{array}{c}\text { Chemisorbed } \\
\text { water }\end{array}$ \\
\hline \multicolumn{7}{c}{ AS-PREPARED Y-AN carbon } \\
\hline Position [eV] & 400.7 & 403.6 & 398.5 & 532.6 & 530.7 & 536.2 \\
Atomic concentration (at. \%) & 2.3 & 0.6 & 2.3 & 4.3 & 1.1 & 1.0 \\
Relative surface concentration (\%) & 45 & 13 & 44 & 67 & 16 & 16 \\
\hline \multicolumn{7}{c}{ Y-AN carbon AFTER 10 } & CYCLES \\
\hline Position [eV] & 400.7 & 403.6 & 398.5 & 532.6 & 530.7 & 536.2 \\
Atomic concentration (at. \%) & 2.6 & 0.6 & 2.4 & 7.6 & 0.7 & 1.2 \\
Relative surface concentration (\%) & 46 & 11 & 43 & 79 & 8 & 13 \\
\hline \multicolumn{7}{c}{ AS-PREPARED Y-Ac carbon } \\
Position [eV] & - & - & - & 532.6 & 530.7 & 536.7 \\
Atomic concentration (at. \%) & - & - & - & 3.84 & 0.33 & 0.91 \\
Relative surface concentration (\%) & - & - & - & 75 & 18 & 6 \\
\hline
\end{tabular}




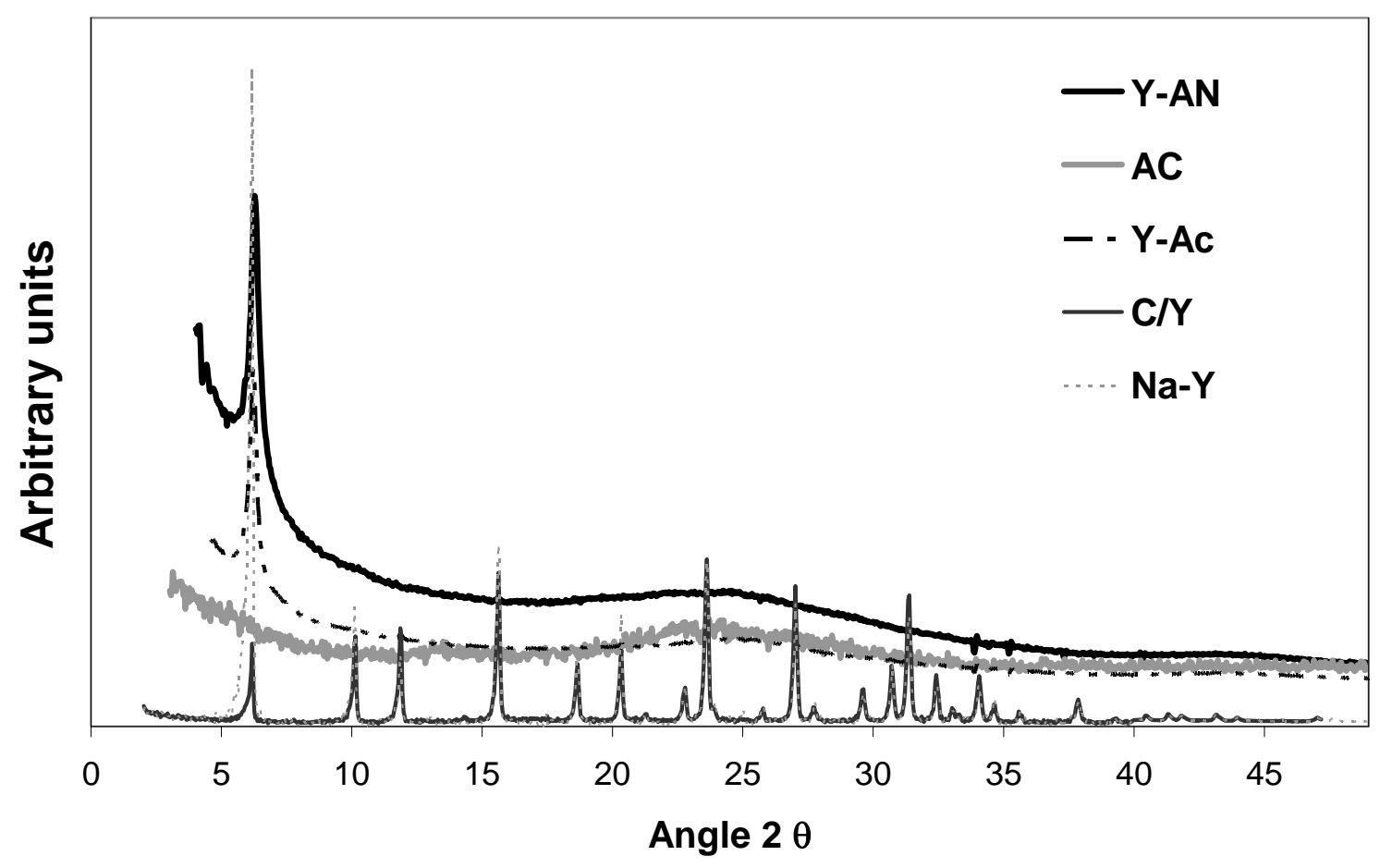

Figure 1. XRD patterns of the inorganic host (Na-Y), the carbon/zeolite composite $(\mathrm{C} / \mathrm{Y})$, the template $(\mathrm{Y}-\mathrm{AN}, \mathrm{Y}-\mathrm{Ac})$ and the commercial $(\mathrm{AC})$ carbon $\left(\mathrm{Cu} \mathrm{K} \alpha_{1}\right.$ radiation $\lambda=0.15405 \mathrm{~nm})$. 




Figure 2. Nitrogen adsorption isotherms at $-196{ }^{\circ} \mathrm{C}$ of the template (Y-AN, Y-Ac) and the commercial (AC) carbons. 


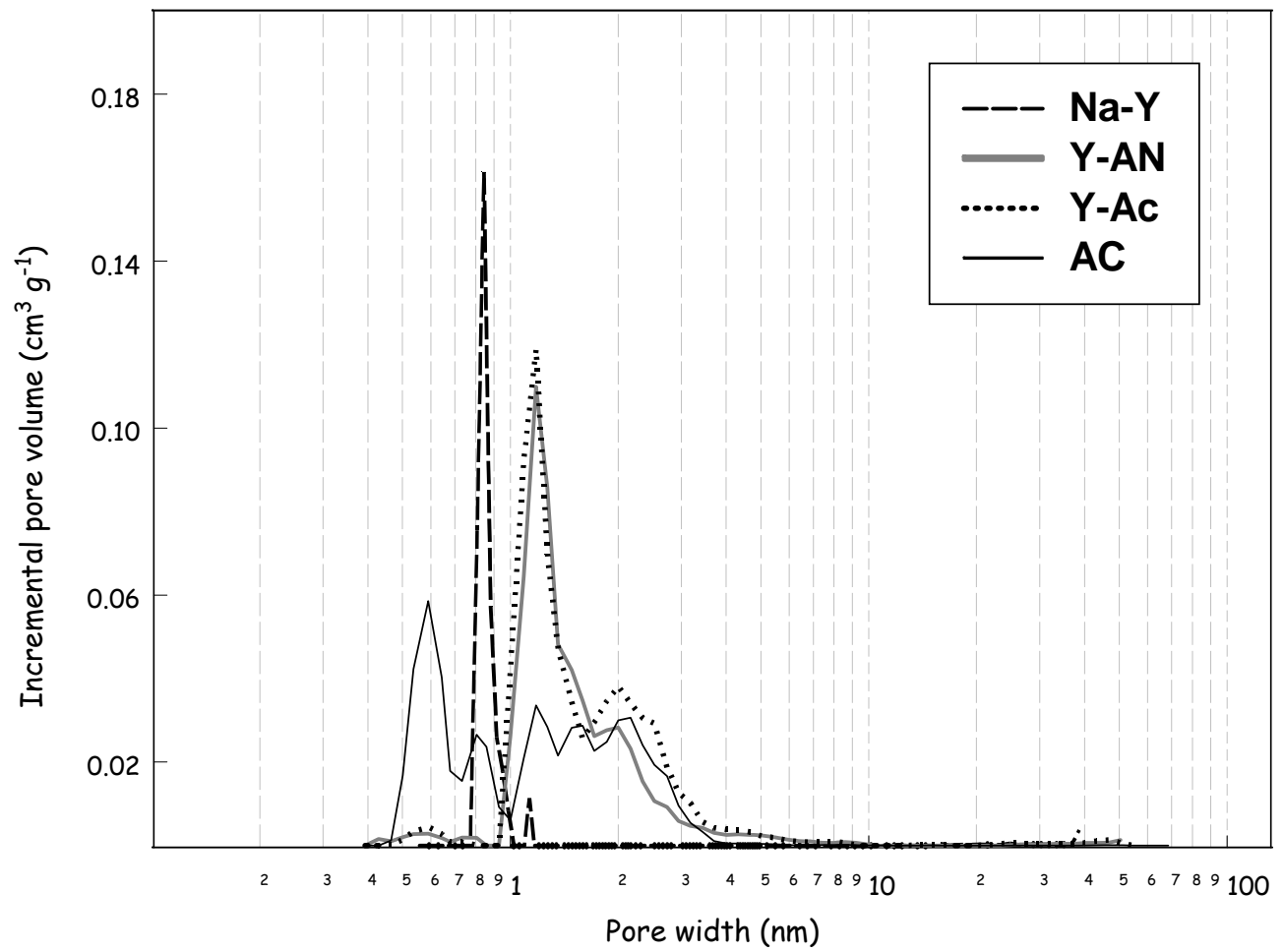

Figure 3. DFT pore size distribution of the template (Y-AN and Y-Ac) and commercial (AC) carbons, along with that of the zeolite (Na-Y) used as host. 


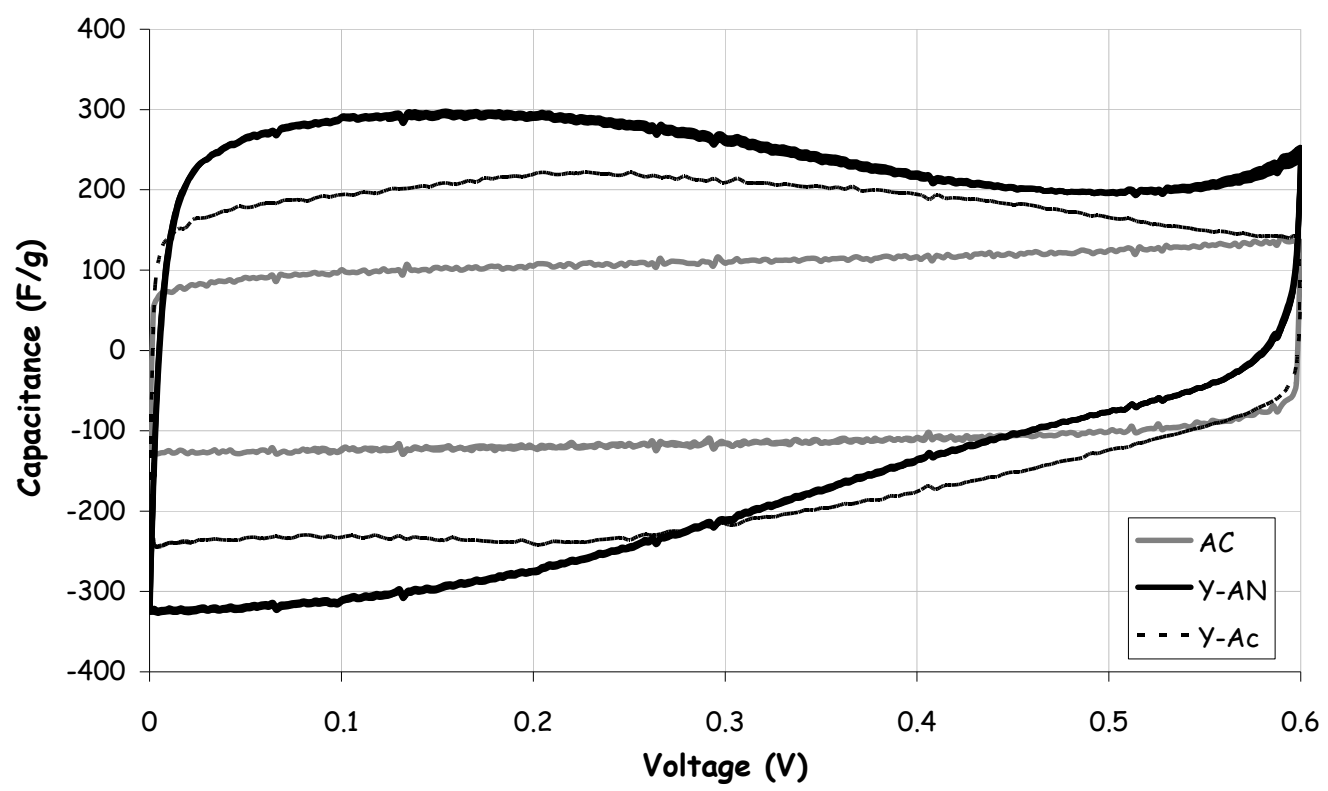

Figure 4. Cyclic voltammograms of two-electrode capacitors built in 1 mol.L ${ }^{-1} \mathrm{H}_{2} \mathrm{SO}_{4}$ from the template carbons Y-AN (solid black line), Y-Ac (dotted black line) and the commercial activated carbon AC (grey line). Scan rate $2 \mathrm{mV} . \mathrm{s}^{-1}$. 


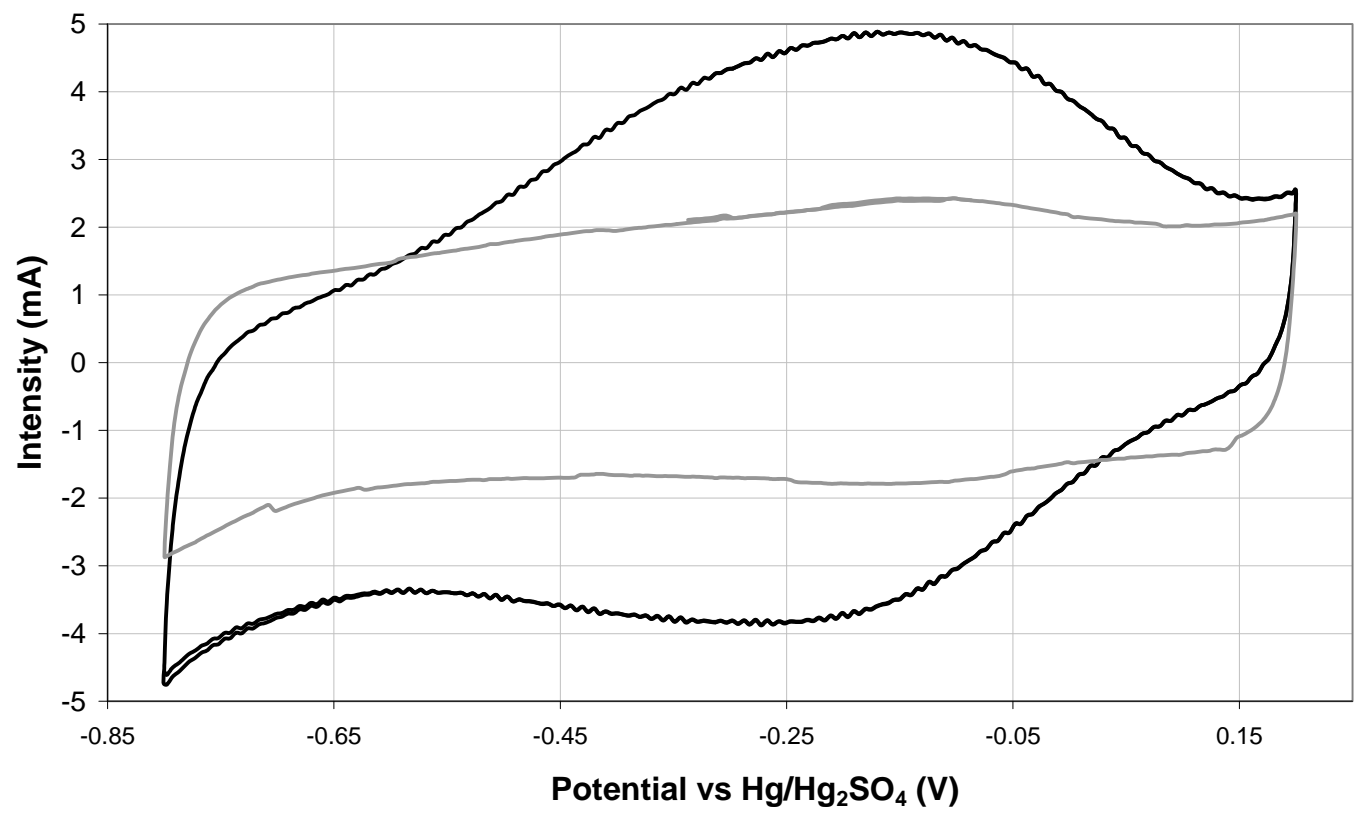

Figure 5. Cyclic voltammograms using a three-electrode cell in $1 \mathrm{~mol} \mathrm{~L}^{-1} \mathrm{H}_{2} \mathrm{SO}_{4}$ for the template carbon Y-AN (black line) and the commercial activated carbon AC (grey line). The counter electrode used was a graphite rod. Reference: $\mathrm{Hg} / \mathrm{Hg}_{2} \mathrm{SO}_{4}$. Scan rate: $2 \mathrm{mV} \mathrm{s}^{-1}$. Mass of pellet: $7 \mathrm{mg}$ in both cases. 


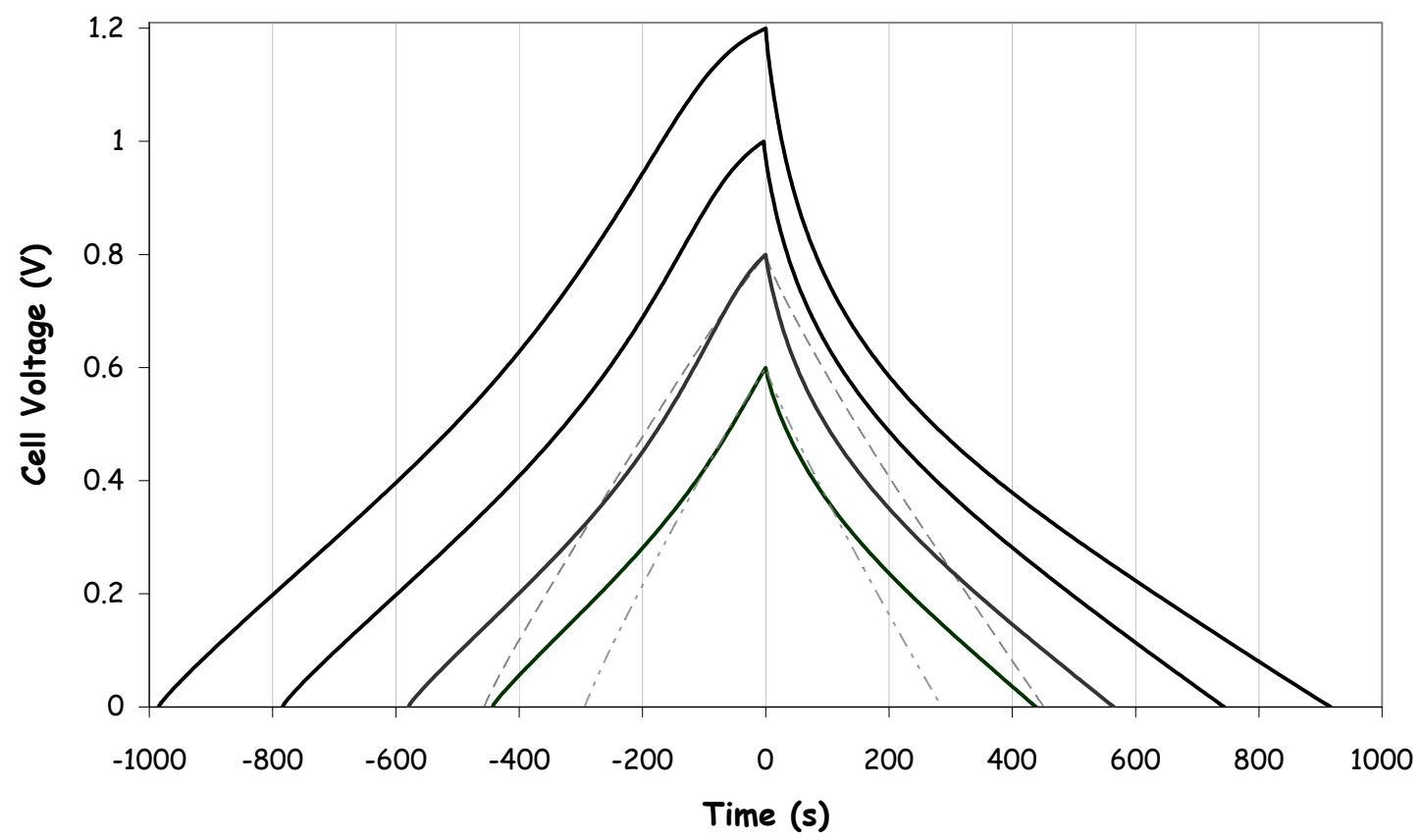

Figure 6. Galvanostatic charge/discharge characteristics of capacitors built from Y-AN (solid line) and AC (dashed line) electrodes at different values of maximum cell voltage. Current load: $100 \mathrm{~mA} \mathrm{~g}^{-1}$; electrolyte: $1 \mathrm{~mol} \mathrm{~L}^{-1} \mathrm{H}_{2} \mathrm{SO}_{4}$. 


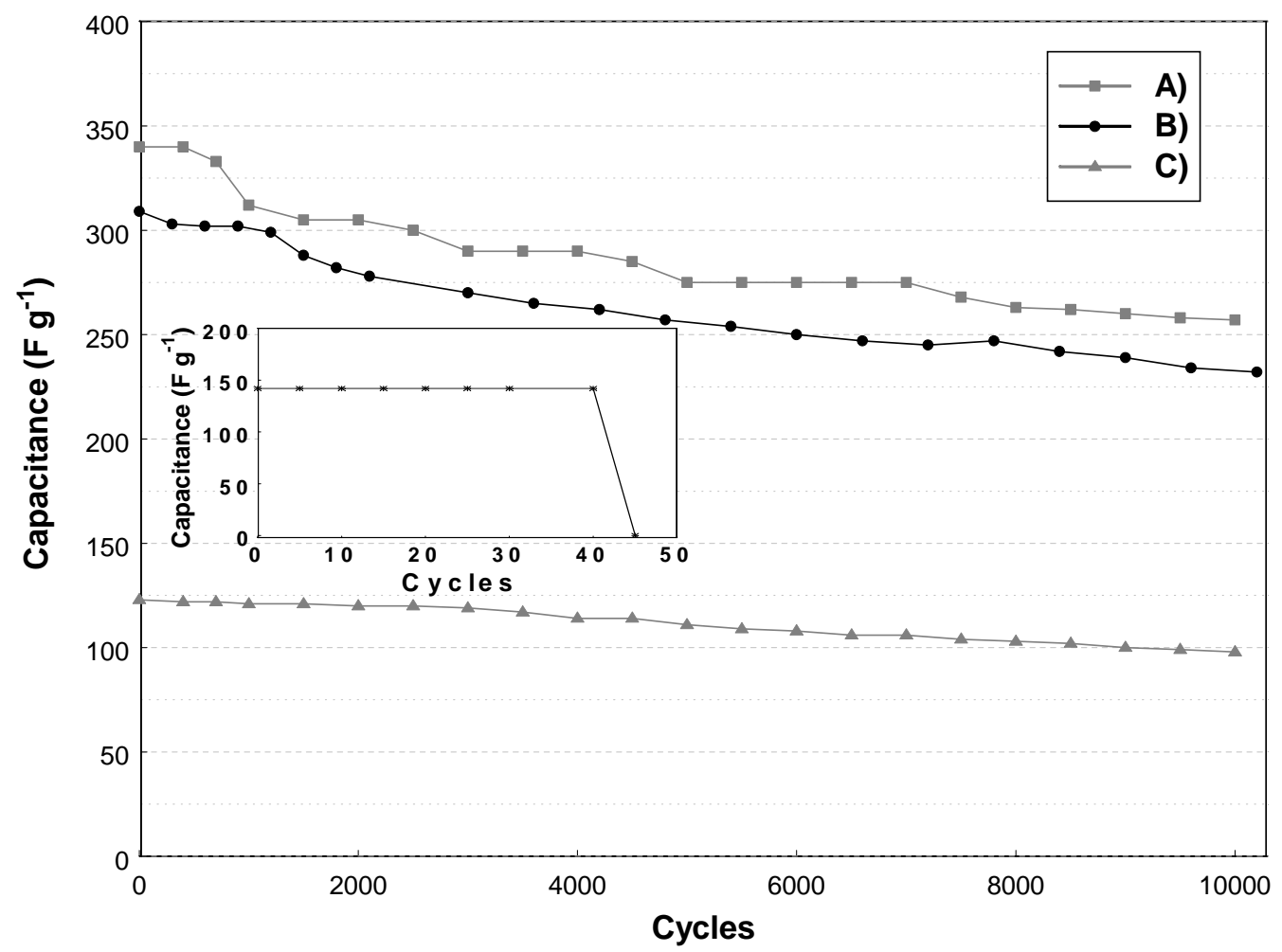

Figure 7. Evolution of the specific discharge capacitance in $1 \mathrm{~mol} \mathrm{~L}^{-1} \mathrm{H}_{2} \mathrm{SO}_{4}$ electrolyte vs the number of cycles: A) template carbon Y-AN with a cell voltage of $0.6 \mathrm{~V}$ and a current density of $100 \mathrm{~mA} \mathrm{~g}^{-1}$; B) template carbon Y-AN with a cell voltage of $1.2 \mathrm{~V}$ and a current density of $200 \mathrm{~mA} \mathrm{~g}^{-1}$; C) commercial carbon AC with a cell voltage of $0.6 \mathrm{~V}$ and a current density of $100 \mathrm{~mA} \mathrm{~g}^{-1}$. Inset: performance of AC at $1 \mathrm{~V}$. 